— Der besonders hohe Aufwand ist dann gegeben, wenn längere Krankheitsverläufe mit mehreren Diagnosen einschließlich des Heraussuchens der Befunde einen Zeitaufwand von deutlich mehr als einer halben Stunde erfordern (iww, Ärztebrief 6/1994).

Allen Einschätzungen gemeinsam ist die Beurteilung, dass zur Erstellung des Gutachtens nicht nur die intellektuelle Arbeit des Arztes, sondern auch die Vorund Nacharbeiten gerechnet werden. Dazu gehören das Heraussuchen der entsprechenden Patientenunterlagen sowie auch letztlich die Erstellung des Gutachtens in Schriftform. Bei Patienten, die seit Jahrzehnten in regelmäßiger Behandlung mit unter Umständen zahlreichen Kontakten sind, und bei denen eine Vielzahl von Fremdbefunden existiert, kann allein die Vorarbeit schon zeitraubend sein. Bei der Gutachtenformulierung muss außerdem Präzision walten, da andernfalls abhängig vom Auftraggeber und der Fragestellung unter Umständen existenzielle Probleme des Patienten die Folge sein können.

\begin{tabular}{|c|c|c|c|c|c|}
\hline $\begin{array}{l}\text { GOÄ } \\
\text { GOP }\end{array}$ & Leistung & Punkte & $x 1,0$ & $x 2,3$ & $x 3,5$ \\
\hline 80 & Schriftliche gutachterliche Äußerung & 300 & $17,49 €$ & $40,22 €$ & $61,20 €$ \\
\hline 85 & $\begin{array}{l}\text { Schriftliche gutachterliche Äußerung mit einem } \\
\text { das gewöhnliche Maß übersteigenden Aufwand } \\
\text { - gegebenenfalls mit wissenschaftlicher Begrün- } \\
\text { dung -, je angefangene Stunde Arbeitszeit }\end{array}$ & 500 & $29,14 €$ & $67,03 €$ & $102,00 €$ \\
\hline 95 & Schreibgebühr, je angefangene DIN-A4-Seite & 60 & $3,50 €$ & - & - \\
\hline 96 & Schreibgebühr, je Kopie & 3 & $0,17 €$ & - & - \\
\hline
\end{tabular}

Neben der GOP 85 sind natürlich auch die GOP 95 (Schreibgebühr) und GOP 96 (Schreibgebühr Kopie) abzurechnen. Dasselbe gilt für die Erstellung von Kopien, die in der Regel mit 0,50 € für die ersten 50 Kopien, bei weiteren Kopien mit jeweils 0,15 € in Rechnung gestellt werden können (LG München, I AZ 9 O 5324/08 vom 19.11.2008) (Tab. 1).

Immer dann, wenn zumindest $30 \mathrm{Mi}$ nuten Arbeitszeit inklusive des Heraussuchens alter Befunde im Archiv und der
Befundsichtung vergangen sind, berechtigt dies zur Abrechnung der GOP 85 und der entsprechenden Schreibgebühren. Eine Faktorensteigerung mit der Begründung „übermäßiger Zeitaufwand“ erübrigt sich, da die Leistung mehrfach berechenbar ist. Eine Steigerung ist allerdings möglich, wenn komplizierte oder mehrere Fragestellung gleichzeitig begutachtet werden sollen.

Dr. med. Heiner Pasch

\title{
GOP 2 GOÄ nicht vergessen
}

\section{$1.260 €$ Umsatzverlust pro Jahr - um soviel geht es, wenn zweimal am Tag die GOP 2 GOÄ vergessen wird. Geld, auf das nicht verzichtet werden muss.}

\footnotetext{
n Zeiten unzähliger Honorardebatten zählt jeder Euro und bekannterweise summiert sich Umsatz oftmals auch aus vielen kleinen Beträgen. Die Bedeutung der GOP 2 GOÄ ist dabei nicht unerheblich, auch wenn das Honorar für diese Leistung bei Abrechnung mit dem Schwellenwert (1,8-fach) lediglich 3,15€ ausmacht. Die GOP 2 ist nicht neben anderen Leistungen beim selben Arzt-Patienten-Kontakt abrechenbar, wohl aber am selben Tag, wenn es sich um zwei unterschiedliche Kontakte handelt, etwa um einen persönlichen Arzt-PatientenKontakt vormittags und eine telefonische Befundmitteilung durch die MFA nachmittags. Die GOP 2 umfasst die - Ausstellung von Wiederholungsrezepten ohne Arztkontakt und/oder _Ausstellung einer Überweisung ohne Arztkontakt und/oder
}

_Übermittlung von Befunden durch die MFA - auch telefonisch und/oder _Übermittlung von ärztlichen Anordnungen durch die MFA - auch telefonisch und/oder

_Messung von Körperzuständen, zum Beispiel Fieber, Blutdruck

Vor allem in großen Praxen führt nicht jedes Wiederholungsrezept oder jede Befundmitteilung durch die MFA auch zu einem Arztkontakt. Ebenso kommen Überweisungen in der PKV vor, auch ohne offizielles Überweisungsformular: Eine entsprechende Notiz auf einem Privatrezept für den Kollegen ist eine Überweisung. Auch die Blutdruckmessung durch die MFA ohne Arztkontakt ist ein abrechnungsfähiger Leistungsbestandteil der GOP 2. Vor allem in Facharztpraxen gehört diese Leistung nicht zur Routine und so wird die Abre- chenbarkeit oft vergessen. Des Weiteren ist die Mitteilung eines Laborwertes am Nachmittag nach Blutabnahme am Vormittag mit entsprechender Uhrzeitangabe als Leistung abrechenbar, ebenso die Anordnungen des Arztes an die MFA, wenn Verhaltenshinweise bis zum später geplanten Sprechstundentermin übermittelt werden. Als Beispiel seien die Lithium-Bestimmung oder das Blutbildergebnis unter laufender Clozapin-Therapie erwähnt (sorgfältige Dokumentation dieser Anordnungen ist erforderlich). Aber auch Anordnungen an späteren Tagen sollten nicht vergessen werden, abzurechnen. Es ist leicht vorstellbar, dass die GOP 2 in der Hektik oder auch aus Unkenntnis nicht abgerechnet wird. Ein Verlustgeschäft, denn wird an jedem Arbeitstag zweimal vergessen, die GOP 2 abzurechnen, ergibt das bei 200 Arbeitstagen im Jahr $200 \times 2 \times 30$ Punkte = 12.000 Punkte gesamt. Bei einem Punktwert von $0,05829 €$ und einem Multiplikator von 1,8 errechnet sich daraus ein Honorarverlust von $12.000 \times 0,05829 \mathrm{x}$ $1,8=1.260 € . \quad$ Dr. med. Heiner Pasch 